\title{
Effect of miR-144-5p on the proliferation, migration, invasion and apoptosis of human umbilical vein endothelial cells by targeting RICTOR and its related mechanisms
}

\author{
WEI FU, ZIDONG LIU, JING ZHANG, YUXUE SHI, RUIYAO ZHAO and HENG ZHAO \\ Department of Cardiology, The Third Affiliated Hospital of Jinzhou Medical University, \\ Jinzhou, Liaoning 121000, P.R. China
}

Received March 19, 2019; Accepted October 08, 2019

DOI: $10.3892 /$ etm.2019.8369

\begin{abstract}
The purpose of the present study was to investigate the effect of microRNA (miR)-144-5p on human umbilical vein endothelial cells (HUVECs) to explore the role of miR-144-5p in atherosclerosis. miR-144-5p expression was upregulated in HUVECs using miR-144-5p mimics. The relative expression level of miR-144-5p in HUVECs was detected using reverse transcription-quantitative PCR (RT-qPCR). Cell proliferation was detected by performing an MTT assay. Apoptosis was determined via flow cytometry. Cell migration ability was detected by a wound-healing assay. Cell invasion was determined by a transwell assay. The protein levels of phosphorylated (p)-PI3K, p-Akt and endothelial nitric oxide synthase (eNOS) were detected using western blot analysis. The binding sites between miR-144-5p and 3'-untranslated region of rapamycin-insensitive companion of mTOR (RICTOR) mRNA were predicted by TargetScan and confirmed by a dual luciferase reporter assay. The present study showed that miR-144-5p mimics significantly inhibited cell proliferation and induced apoptosis in HUVECs. In addition, miR-144-5p mimics could suppress migration and invasion of HUVECs. Further analysis identified that RICTOR was a direct target gene of miR-144-5p. Moreover, miR-144-5p upregulation decreased the protein level of p-PI3K, p-Akt and eNOS. In conclusion, miR-144-5p regulated HUVEC proliferation, migration, invasion, and apoptosis through affecting the PI3K-Akt-eNOS signaling pathway by altering the expression of RICTOR. These results indicated that miR-144-5p may be a potential target for the prevention and treatment of atherosclerosis.
\end{abstract}

Correspondence to: Dr Zidong Liu, Department of Cardiology, The Third Affiliated Hospital of Jinzhou Medical University, 2 Heping Road, Linghe, Jinzhou, Liaoning 121000, P.R. China E-mail: liuzd181201@163.com

Key words: microRNA-144, rapamycin-insensitive companion of mTOR, human umbilical vein endothelial cells, migration, invasion

\section{Introduction}

Atherosclerosis is a chronic inflammatory and vascular disease, which frequently causes heart disease and stroke (1). Atherosclerosis is involved in the accumulation of lipid-engorged macrophages in the subendothelial layer of arterial vessels $(2,3)$. Endothelial dysfunction is one of the main contributors to atherosclerosis (4) and results in a phenotypic alteration that increases the expression of adhesion molecules released by endothelial cells (ECs). Apoptosis of endothelial cells plays an important role in the occurrence and development of atherosclerosis (5).

MicroRNAs (miRNAs/miRs) are a group of small, single-stranded, non-coding RNA molecules which influence the synthesis of proteins via their interactions with target mRNAs $(6,7)$. It has been reported that miRNAs could modulate a number of biological processes, including tumorigenesis, development, invasion, metastasis and other biological characteristics $(8,9)$. Accumulating evidence suggests that miRNAs serve as diagnostic and prognostic biomarkers of numerous types of cancer $(10,11)$. Some miRNAs have been reported to be involved in atherosclerosis through regulating vascular cells (12). Previous research indicated that miRNAs were closely related to the pathogenesis of atherosclerosis $(13,14)$.

Previous studies reported that several miRNAs, such as miR-34a, miR-217, and miR-146a, could regulate the proliferation and differentiation of ECs and may also stimulate cellular senescence, which can act as a trigger for endothelial dysfunction (15-17). miR-144 is a conservative miRNA and tumor suppressor gene that plays a role in tumor suppression in a variety of cancers, such as bladder cancer (18), thyroid cancer (19), gastric cancer (20), colorectal cancer (21), ovarian cancer (22) and nasopharyngeal carcinoma (NPC) (23). Moreover, previous studies reported that miR-144 upregulation may inhibit the proliferation, invasion and metastasis of cancer cells $(21,24)$. However, the specific function and mechanism of miR-144-5p in atherosclerosis remain unclear. Apoptosis of endothelial cells plays an important role in the occurrence and development of atherosclerosis. Therefore, the present study aimed to investigate the effects of miR-144-5p 
on human umbilical vein endothelial cells (HUVECs) to explore the role of miR-144-5p in atherosclerosis.

\section{Materials and methods}

Cell culture. HUVECs were obtained from Shanghai Institute of Life Sciences, Chinese Academy of Sciences. HUVECs were cultured in medium 199 supplemented with 10\% FBS, $1 \%$ penicillin and streptomycin (Gibco; Thermo Fisher Scientific, Inc.), endothelial cell growth supplement (Sigma-Aldrich; Merck KGaA) and epidermal growth factor (10 ng/ml, Roche Diagnostics) in a humidified atmosphere at $37^{\circ} \mathrm{C}$ with $5 \% \mathrm{CO}_{2}$.

Cell transfection. Once cells reached 70-80\% confluency, miR-144-5p mimics (Guangzhou RiboBio Co., Ltd.) or the negative control of miR-144-5p mimics (mimics-NC; Guangzhou RiboBio Co., Ltd.) were transfected into HUVECs using Lipofectamine ${ }^{\circledR} 2000$ (Invitrogen; Thermo Fisher Scientific Inc.) according to the manufacturer's protocols. The transfection efficiency was detected by reverse transcription-quantitative PCR (RT-qPCR), as described below.

Flow cytometry analysis. After transfection, HUVECs were washed three times with PBS and centrifuged at $1,000 \times \mathrm{g}$ for $5 \mathrm{~min}$ at $20^{\circ} \mathrm{C}$. Harvested cells were stained with $200 \mu \mathrm{l}$ annexin V/FITC binding buffer (Beyotime Institute of Biotechnology) and then incubated with $5 \mu 1$ propidium iodide (PI; Beyotime Institute of Biotechnology) for $25 \mathrm{~min}$ at room temperature without light. Cell apoptosis was analyzed by flow cytometry (BD FACSCalibur ${ }^{\mathrm{TM}}$; Becton, Dickinson and Company) and data were analyzed using FlowJo software (version 7.6.1; FlowJo LLC). Each experiment was performed in triplicate.

Cell invasion assay. Cell invasion ability was detected using a Transwell chamber (pore size, $8 \mu \mathrm{m}$; Costar; Corning Inc.) pre-coated with Matrigel $\left(1 \mathrm{mg} / \mathrm{ml}\right.$; BD Biosciences) at $4^{\circ} \mathrm{C}$ overnight. HUVECs $\left(2 \times 10^{4}\right.$ cells/well) were briefly plated into the upper chambers using human endothelial serum-free medium (cat. no. 11111044; Gibco; Thermo Fisher Scientific, Inc.) containing $0.5 \%$ FBS. Medium (DMEM; Gibco; Thermo Fisher Scientific, Inc.) containing 10\% FBS was added to the lower chamber as a chemoattractant, stimulating cells to move to the lower chamber. At $24 \mathrm{~h}$ after incubation, the invasive cells on the lower chamber were fixed with $4 \%$ paraformaldehyde at room temperature for $30 \mathrm{~min}$. Subsequently, the membrane was stained for $30 \mathrm{~min}$ with $0.1 \%$ crystal violet at room temperature. Cells located on the top of membrane were wiped with a cotton swab. The Transwell chamber was then washed with $95 \%$ ethanol. A total of 10 fields of view were selected randomly to count the number of cells under a light microscope (magnification, x200; Nikon Corporation). Each group was repeated in triplicate and all experiments were repeated three times to obtain average values.

Cell migration assay. A wound-healing assay was performed to detect cell migration ability. HUVECs $\left(3 \times 10^{5}\right.$ cells/well) were seeded in six-well plates. Cells were grown until $90 \%$ confluency before each well was scraped with a $10 \mu \mathrm{l}$ pipette tip to create a linear region devoid of cells. Subsequently, the cells were washed with PBS three times. Human endothelial serum-free culture medium (cat. no. 11111044; Gibco; Thermo Fisher Scientific, Inc.) was then added to cells and incubated at $37^{\circ} \mathrm{C}$ with $5 \% \mathrm{CO}_{2}$. Wound healing was monitored using a light microscope (magnification, x100; Nikon Corporation) at 0 and $24 \mathrm{~h}$ after scraping. Percentage of wound $(\%)=($ Wound width at $24 \mathrm{~h} /$ Wound width at $0 \mathrm{~h}) \times 100$.

MTT assay. HUVECs were inoculated in a 96-well plate at a density of $1 \times 10^{4}$ cells/well at $37^{\circ} \mathrm{C}$ with $5 \% \mathrm{CO}_{2}$ for 12,24 and $48 \mathrm{~h}$. Subsequently, MTT ( $20 \mu \mathrm{l} ; 5 \mathrm{mg} / \mathrm{ml})$ was added to each well, and cells were incubated for $4 \mathrm{~h}$. A total of $150 \mu \mathrm{l}$ DMSO was added to dissolve formazan crystals and gently shaken for $10 \mathrm{~min}$. The optical density of each sample was determined with a microplate reader (Multiskan ${ }^{\mathrm{TM}}$ FC; Thermo Fisher Scientific, Inc.) by measuring the absorbance at $490 \mathrm{~nm}$. The experiments were repeated at least three times.

Western blot analysis. Total protein was extracted from HUVECs using radioimmunoprecipitation assay buffer containing $1 \mathrm{mM}$ PMSF. Bicinchoninic Acid Protein assay kit (Thermo Fisher Scientific, Inc.) was then used to quantify protein concentration following which the protein samples were separated by $10 \%$ SDS-PAGE (20 $\mu \mathrm{g} /$ lane) and transferred onto PVDF membranes. Subsequently, the membrane was blocked with $5 \%$ skim milk for $1 \mathrm{~h}$ at room temperature and incubated with primary antibodies against phosphorylated (p)-AKT (cat. no. ab81283; 1:1,000; Abcam), AKT (cat. no. ab179463, 1:10,000, Abcam), p-PI3K (cat. no. ab182651, 1:1,000, Abcam), PI3K (cat. no. ab86714, 1:1,000, Abcam), eNOS (cat. no. ab66127, 1:1,000, Abcam) or GAPDH (cat. no. ab181602, 1:10,000, Abcam) at $4^{\circ} \mathrm{C}$ overnight. The membrane was then incubated with horseradish peroxidase-conjugated secondary antibody Goat Anti-Rabbit IgG H\&L (ab205718; 1:2,000; Abcam), Goat Anti-Mouse IgG H\&L (ab205719; 1:2,000; Abcam) and for $2 \mathrm{~h}$ at room temperature. The GAPDH was used as internal reference. Afterwards, the protein bands were detected and visualized using an enhanced chemiluminescence method (RapidStep ${ }^{\mathrm{TM}}$ ECL Reagent; Merck KGaA). The densities of bands were semi-quantitively analyzed using ImageJ (version 4.0; National Institutes of Health).

RT-qPCR assay. Total RNA was extracted from HUVECs using TRIzol ${ }^{\circledR}$ reagent (Invitrogen; Thermo Fisher Scientific, Inc.) following the manufacturer's protocols. Total RNA concentration was detected by NanoDrop ${ }^{\mathrm{TM}} 2000$ (Thermo Fisher Scientific, Inc.) and RNA samples were subsequently stored at $-80^{\circ} \mathrm{C}$. cDNA synthesis was performed using the RevertAid $^{\text {TM }}$ First Strand cDNA Synthesis kit (Thermo Fisher Scientific, Inc.). The temperature protocol was as follows: $42^{\circ} \mathrm{C}$ for $30 \mathrm{~min}$ and $85^{\circ} \mathrm{C}$ for $5 \mathrm{~min}$. RT-qPCR was performed using a QuantiFast SYBR Green PCR kit (Qiagen GmbH) in accordance with the manufacturer's protocols. The following primers were used: U6 forward, 5'-GCTTCGGCAGCACAT ATACTAAAAT-3' and reverse, 5'-CGCTTCACGAATTTG CGTGTCAT-3'; miR-144-5p forward, 5'-GCGCGAATTCGA GATCTTAACAGACCCTAGCTC-3' and reverse, 5'-GCG CGGATCCGTGCCCTGGCAGTCAGTAGG-3'. Relative expression levels were calculated using the $2^{-\Delta \Delta C q}$ method (25) 
A

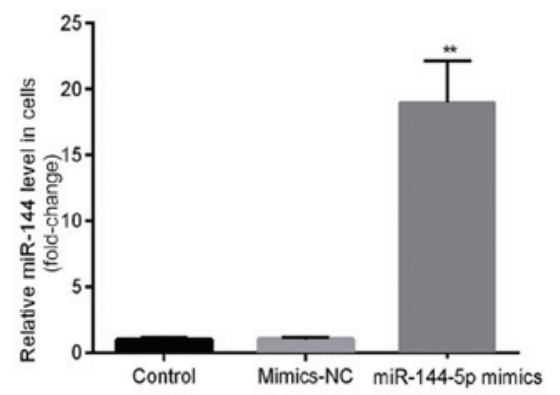

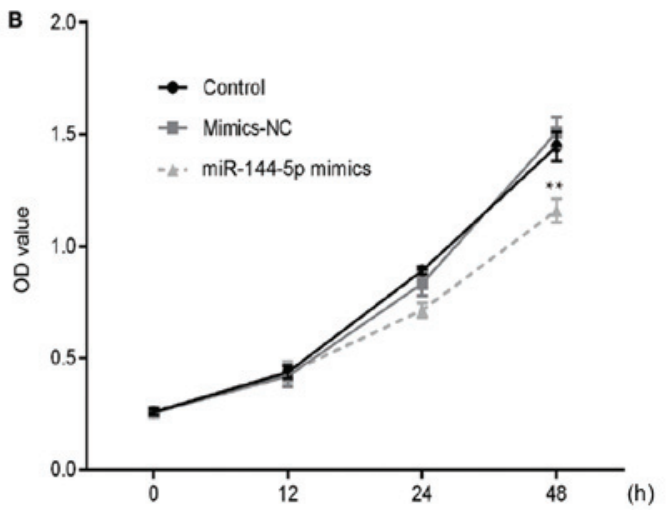

c

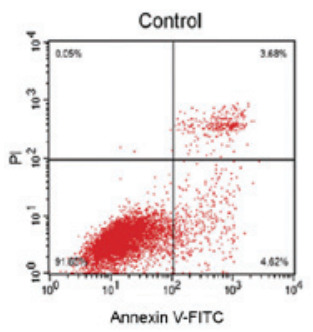

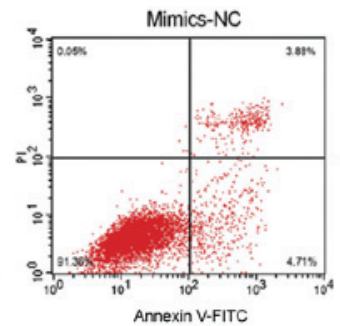
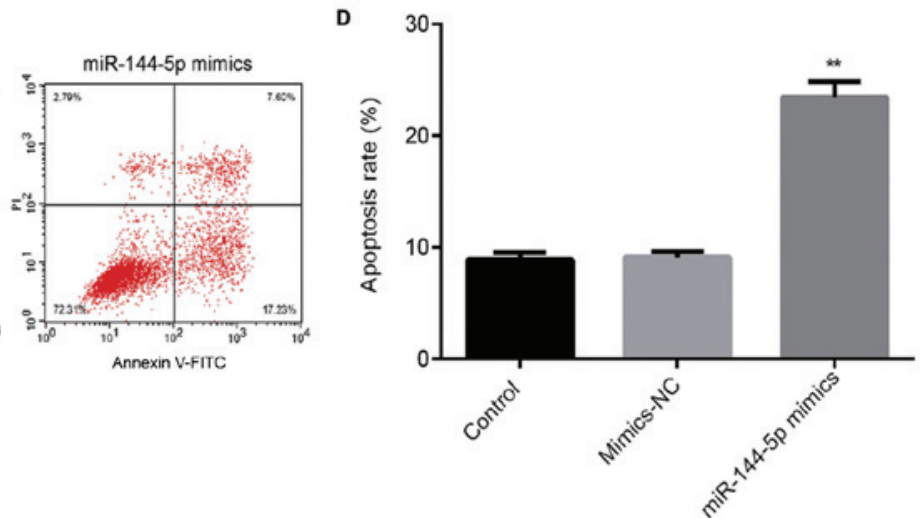

Figure 1. miR-144-5p suppresses cell proliferation and promotes apoptosis. (A) A reverse transcription-quantitative PCR assay detected the relative expression of miR-144-5p in HUVECs transfected with miR-144-5p mimic or mimic-NC for $48 \mathrm{~h}$. (B) An MTT assay was performed to determine the proliferation in HUVECs transfected with miR-144-5p mimics or mimics-NC for $48 \mathrm{~h}$. (C) A flow cytometry assay was performed to determine apoptosis in HUVECs transfected with miR-144-5p mimics or mimics-NC for 48 h. (D) Apoptotic rate of transfected cells compared with non-transfected cells. ${ }^{* *} \mathrm{P}<0.01$ vs. mimic-NC. NC, negative control; miR, microRNA.

after normalization with reference to U6 expression. All experiments were performed in triplicate.

Dual-luciferase reporter assay. TargetScan 7.2 (http://www. targetscan.org/vert_72/) was used to predict the potential targets of miR-144-5p. The results showed the binding sites between miR-144-5p and the RICTOR 3'-untranslated region (UTR). Subsequently, a dual-luciferase reporter assay was performed to confirm the predicted binding sites. According to the bioinformatics analysis results, a fragment of the RICTOR 3'-UTR containing the wild type (WT) or mutant (5'-AGAAGAGGUUAGGAACACCCCGA-3') seed regions of miR-144-5p was chemically synthesized in vitro and cloned into BamHI and AscI sites of the pMIR-RB-REPORT ${ }^{\mathrm{TM}}$ vector (Guangzhou RiboBio Co., Ltd.). HUVECs were then co-transfected with miR-144-5p mimics or mimics-NC and WT-RICTOR or Mutant-RICTOR with Lipofectamine 2000 for $48 \mathrm{~h}$. After a 48-h incubation, relative luciferase activity was calculated by normalizing to Renilla luciferase activity using the Dual Luciferase Reporter Assay System (Promega Corporation) following the manufacturer's protocol.

Statistical analysis. All data were are presented as the mean \pm standard deviation. All experiments were performed at least three times. SPSS 19.0 software (IBM Corp.) was used to perform data analysis. Comparisons between groups were analyzed using Student's t-test and one-way analysis of variance followed by Tukey's post hoc test. $\mathrm{P}<0.05$ was considered to indicate a statistically significant difference.

\section{Results}

Effect of miR-144-5p on cell proliferation and apoptosis of HUVECs. An RT-qPCR assay was first performed to detect the transfection efficiency of miR-144-5p. RT-qPCR results showed that miR-144-5p mimics significantly increased the relative expression of miR-144-5p in HUVECs compared with the mimic-NC (Fig. 1A). To investigate the effects of miR-144-5p on HUVEC proliferation and apoptosis, MTT and flow cytometry assays were performed. MTT assay results demonstrated that miR-144-5p mimics significantly reduced cell proliferation compared to the mimic-NC group (Fig. 1B). Flow cytometry results indicated that miR-144-5p mimics significantly promoted cell apoptosis of HUVECs compared with the mimic-NC group (Fig. 1C and D).

Effect of miR-144-5p on cell migration and invasion of HUVECs. In order to investigate the migration and invasion of cells in different groups, wound healing and transwell assays were performed. Results from the wound healing assay revealed that miR-144-5p mimics significantly inhibited the cell migration ability of HUVECs compared with 
A
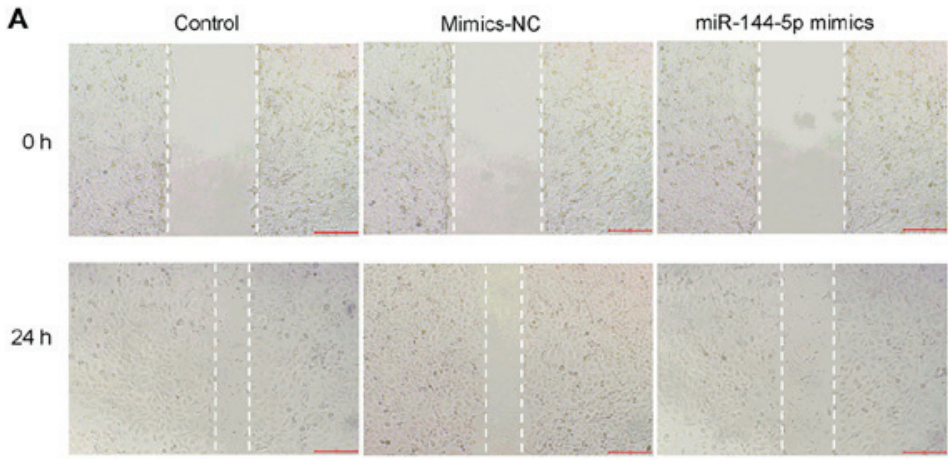

C

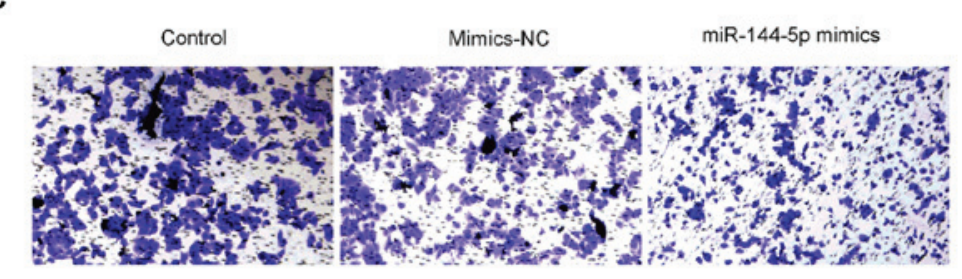

B



D

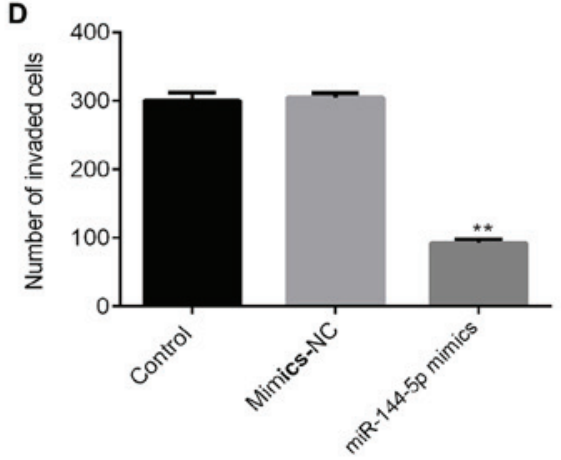

Figure 2. miR-144-5p mimics suppress cell migration and invasion of HUVECs. (A) A wound healing assay was performed to determine cell migration in HUVECs transfected with miR-144 mimic or mimic-NC for $24 \mathrm{~h}$. Scale bars, $50 \mu \mathrm{m}$. (B) Percentage of wound data are presented as the mean $\pm \mathrm{SD}$ from three independent experiments. (C) A Transwell assay was performed to determine cell invasion in HUVECs transfected with miR-144-5p mimics or mimic-NC for $24 \mathrm{~h}$ (magnification, $\mathrm{x} 200$ ). (D) The number of invaded cells was calculated and presented as the mean $\pm \mathrm{SD}$ from three independent experiments. ${ }^{* *} \mathrm{P}<0.01 \mathrm{vs}$. mimic-NC. NC, negative control; miR, microRNA.

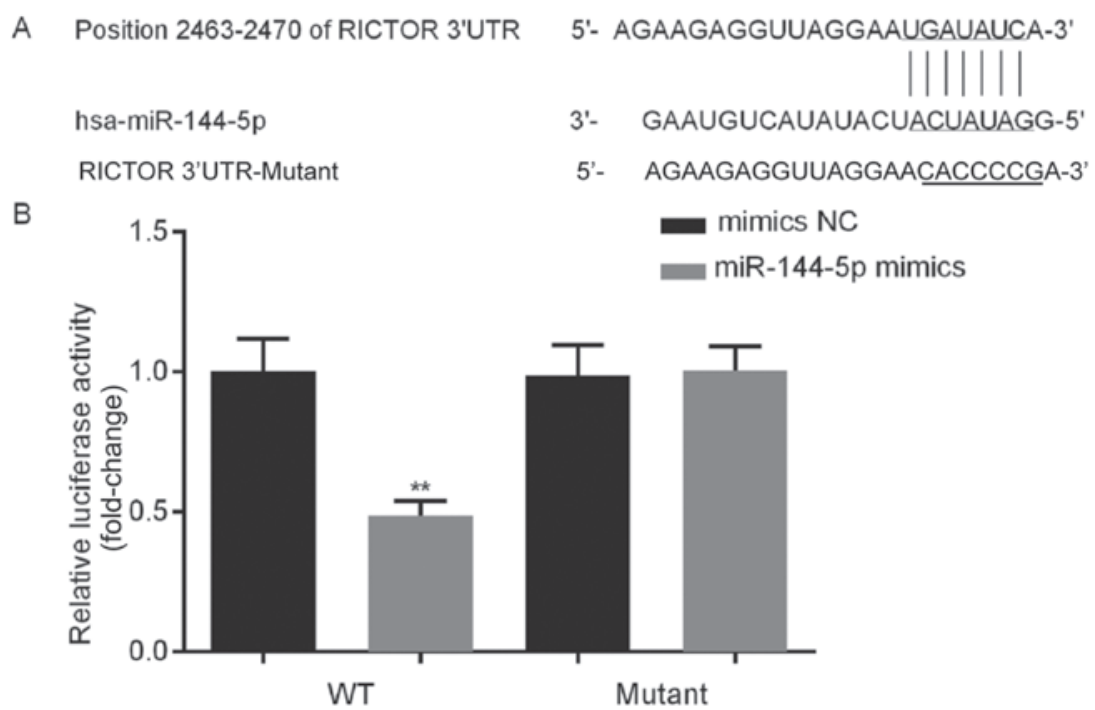

Figure 3. RICTOR is a direct target gene of miR-144-5p. (A) Binding sites between miR-144-5p and RICTOR. (B) A dual-luciferase reporter assay was used to confirm the binding sites between miR-144-5p and RICTOR in HUVECs. ${ }^{* *} \mathrm{P}<0.01$ vs. mimic-NC. RICTOR, rapamycin-insensitive companion of mTOR; UTR, untranslated region; NC, negative control; miR, microRNA; WT, wild-type.

the mimic-NC group (Fig. 2A and B). The transwell assay results showed that miR-144-5p mimics significantly reduced the invasive capacity and decreased the number of invasive HUVECs (Fig. 2C and D).

RICTOR is a direct target gene of miR-144-5p. To investigate the potential role of miR-144-5p in the growth of HUVECs, bioinformatics analysis was performed to predict the potential targets of miR-144-5p. The results identified binding sites between the 3'-UTR of RICTOR and miR-144-5p (Fig. 3A). A dual-luciferase reporter system was used to determine if RICTOR was a direct target of miR-144-5p. The results indicated that compared with the relative luciferase activity of cells co-transfected with WT RICTOR 3'-UTR and mimics-NC, 
A

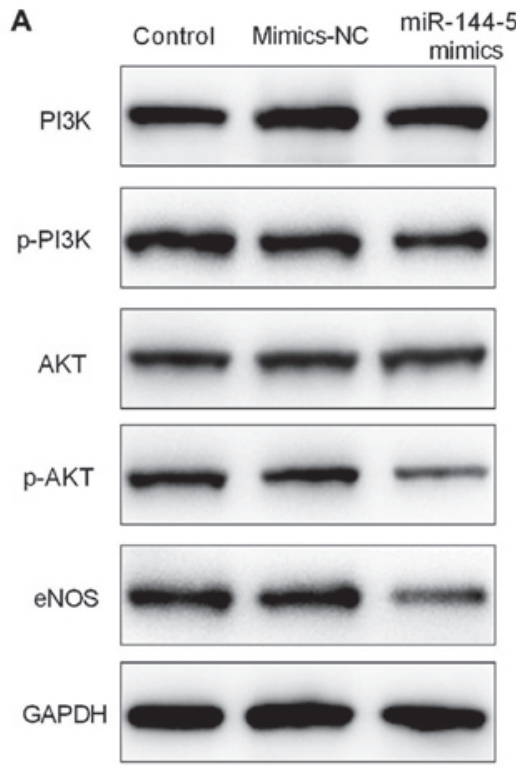

B

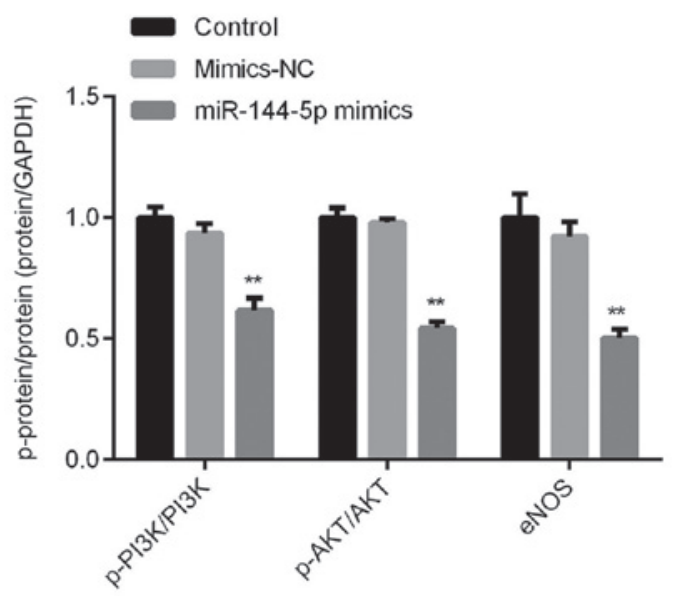

Figure 4. miR-144-5p mimics inhibit the protein levels of p-PI3K, p-Akt and eNOS. (A) Western blot assay detected the protein levels of p-PI3K, PI3K, p-Akt, Akt and eNOS, and GAPDH was used as an internal control. (B) Ratios of p-PI3K/PI3K, p-Akt/Akt, and eNOS/GAPDH were calculated. ${ }^{* *} \mathrm{P}<0.01 \mathrm{vs}$. mimic-NC eNOS, endothelial nitric oxide synthase; NC, negative control; miR, microRNA.

the relative luciferase activity of cells co-transfected with WT RICTOR 3'-UTR and miR-144-5p mimics significantly decreased (Fig. 3B). Furthermore, when comparing the relative luciferase activity of cells co-transfected with mutant RICTOR 3'-UTR and mimics-NC, the relative luciferase activity of cells co-transfected with mutant RICTOR 3'-UTR and miR-144-5p mimics showed no significant changes (Fig. 3B). These data indicated that RICTOR was a direct target gene of miR-144-5p.

miR-144-5p is associated with PI3K-Akt-endothelial nitric oxide synthase (eNOS) pathway in HUVECs. In order to further explore the specific mechanism of the effect of miR-144-5p on HUVECs, the expression levels of related proteins in the PI3K-Akt-eNOS pathway were detected. Results from the western blot analysis indicated that miR-144-5p mimics decreased the protein levels of phosphorylated (p)-PI3K, p-Akt and eNOS in HUVECs (Fig. 4A and B).

\section{Discussion}

Atherosclerosis is a relatively complex disease affecting medium and large-sized arteries. Vascular injury plays and important role in the pathogenesis of atherosclerosis, and endothelial apoptosis plays a crucial role in endothelial dysfunction and atherosclerosis lesion formation (26). Currently, atherosclerosis is being extensively studied. Therefore, the current study aimed to explore the role of miR-144-5p in atherosclerosis development through investigating the effects of miR-144-5p on endothelial cells. miR-144-5p mimics inhibited HUVEC proliferation and promoted cell apoptosis. In addition, miR-144-5p mimics could decrease cell migration and invasion of HUVECs. Moreover, RICTOR was a direct target of miR-144-5p. Finally, miR-144-5p mimics could repress PI3K-Akt-eNOS signaling pathway in HUVECs.

Previously, miRNAs have been confirmed to be involved in numerous important physiological processes (27-30).
miRNAs are vital regulators for endothelial biology and dysfunction. Deregulation of miRNA expression is involved in endothelial angiogenesis, injury, inflammation and senescence (31-33).

miR-144-5p has been studied in various cancers such as renal cell carcinoma (34) and miR-144-5p has also been studied in chronic periodontitis (35). A previous study demonstrated that circulating miR-144-5p was associated with depressive disorders (36). However, the role and mechanism of miR-144-5p in atherosclerosis remains unclear. Apoptosis of endothelial cells plays an important role in the occurrence and development of atherosclerosis (26). The present study investigated the effect of miR-144-5p on HUVECs to explore the role of miR-144-5p in atherosclerosis.

The effect of miR-144-5p upregulation on the biological behaviors of HUVECs was determined by performing MTT, migration and invasion assays, and flow cytometry. The results demonstrated that miR-144-5p mimics significantly inhibited HUVEC proliferation, migration and invasion, and induced cell apoptosis.

Subsequently, to explore the underlying mechanism of miR-144-5p on HUVECs, bioinformatics analysis was used to predict the potential targets of miR-144-5p. RICTOR was a direct target of miR-144-5p in HUVECs. RICTOR is a crucial component of mTOR complex 2 (mTORC2), and the activation of mTORC2 depends on the presence of the RICTOR protein. In addition, RICTOR is required for the inactivating Ser473 phosphorylation on Akt (37). Furthermore, eNOS activity dysfunction leads to a decrease in NO bioavailability, which results in atherosclerosis (38). A previous study revealed that the RICTOR/Akt/eNOS pathway played an important role in regulating apoptosis and dysfunction in EC cells (39). Thus, the present study explored whether the RICTOR/Akt/eNOS pathway was affected by miR-144-5p in HUVECs. The results indicated that miR-144-5p mimics decreased the protein levels of p-PI3K, p-Akt and eNOS in 
HUVECs. However, p-eNOS levels were not detected in the current study, which is a limitation.

In conclusion, miR-144-5p inhibited the proliferation, invasion and migration of HUVECs, and induced apoptosis through regulating the PI3K-Akt-eNOS signaling pathway, at least partly by modulating RICTOR expression. Thus, miR-144-5p might participate in the occurrence and development of atherosclerosis through regulating the apoptosis of ECs. However, the current study only included a preliminary analysis of miR-144-5p in atherosclerosis. To completely elucidate the role of miR-144-5p in atherosclerosis, further in-depth research is needed. For example, the protective effects of RICTOR, PI3K, Akt, and eNOS on HUVECs should be investigated. The role of miR-144-5p in atherosclerosis should be studied in vivo. In addition, the expression of miR-144-5p in patients with atherosclerosis and its relationship with the clinicopathological features of patients can be further explored.

\section{Acknowledgements}

Not applicable.

\section{Funding}

No funding was received.

\section{Availability of data and materials}

The datasets used and/or analyzed during the current study are available from the corresponding author on reasonable request.

\section{Authors' contributions}

ZL designed the study and revised the manuscript. WF and JZ wrote the manuscript and collected the data. YS and RZ searched the literature and interpreted the data. HZ collected the data. All authors read and approved the final manuscript.

\section{Ethics approval and consent to participate}

Not applicable.

\section{Patient consent for publication}

Not applicable.

\section{Competing interests}

The authors declare that they have no competing interests.

\section{References}

1. Lusis AJ: Atherosclerosis. Nature 407: 233-241, 2000.

2. Hansson GK, Libby P and Tabas I: Inflammation and plaque vulnerability. J Intern Med 278: 483-493, 2015.

3. Libby P and Hansson GK: Inflammation and immunity in diseases of the arterial tree: Players and layers. Circ Res 116: 307-311, 2015.

4. Tabas I, García-Cardeña G and Owens GK: Recent insights into the cellular biology of atherosclerosis. J Cell Biol 209: 13-22, 2015

5. Mudau M, Genis A, Lochner A and Strijdom H: Endothelial dysfunction: The early predictor of atherosclerosis. Cardiovasc J Afr 23: 222-231, 2012
6. Thum T and Mayr M: Review focus on the role of microRNA in cardiovascular biology and disease. Cardiovasc Res 93: 543-544, 2012.

7. Winter J, Jung S, Keller S, Gregory RI and Diederichs S: Many roads to maturity: MicroRNA biogenesis pathways and their regulation. Nat Cell Biol 11: 228-234, 2009.

8. Natarelli L and Schober A: MicroRNAs and the response to injury in atherosclerosis. Hamostaseologie 35: 142-150, 2015.

9. Hwang HW and Mendell JT: MicroRNAs in cell proliferation, cell death, and tumorigenesis. Br J Cancer 96: 776-780, 2006.

10. Matsushita R, Seki N, Chiyomaru T, Inoguchi S, Ishihara T, Goto Y, Nishikawa R, Mataki H, Tatarano S, Itesako T, et al: Tumour-suppressive microRNA-144-5p directly targets $\mathrm{CCNE} 1 / 2$ as potential prognostic markers in bladder cancer. Br J Cancer 113: 282-289, 2015.

11. Song L, Peng L, Hua S, Li X, Ma L, Jie J, Chen D, Wang Y and Li D: miR-144-5p enhances the radiosensitivity of non-small-cell lung cancer cells via targeting ATF2. Biomed Res Int 2018: 5109497, 2018

12. Ji R, Cheng Y, Yue J, Yue J, Yang J, Liu X, Chen H, Dean DB and Zhang C: MicroRNA expression signature and antisense-mediated depletion reveal an essential role of microRNA in vascular neointimal lesion formation. Circ Res 100: 1579-1588, 2007.

13. Shan Z, Yao C, Li ZL, Teng Y, Li W, Wang JS, Ye CS, Chang GQ, Huang XL, Li XX, et al: Differentially expressed microRNAs at different stages of atherosclerosis in ApoE-deficient mice. Chin Med J (Engl) 126: 515-520, 2013.

14. Hosin AA, Prasad A, Viiri LE, Davies AH and Shalhoub J: MicroRNAs in atherosclerosis. J Vasc Res 51: 338-349, 2014.

15. Alexandru N, Badila E, Weiss E, Cochior D, Stępień E and Georgescu A: Vascular complications in diabetes: Microparticles and microparticle associated microRNAs as active players. Biochem Biophys Res Commun 472: 1-10, 2016.

16. Kumar S, Kim CW, Simmons RD and Jo H: Role of flow-sensitive microRNAs in endothelial dysfunction and atherosclerosis: Mechanosensitive athero-miRs. Arterioscler Thromb Vasc Biol 34: 2206-2216, 2014.

17. Sun X, Belkin N and Feinberg MW: Endothelial microRNAs and atherosclerosis. Curr Atheroscler Rep 15: 372, 2013.

18. Guo Y, Ying L, Tian Y Yang P, Zhu Y, Wang Z, Qiu F and Lin J: miR-144 downregulation increases bladder cancer cell proliferation by targeting EZH2 and regulating Wnt signaling. FEBS J 280: 4531-4538, 2013.

19. Guan H, Liang W, Xie Z, Li H, Liu J, Liu L, Xiu L and Li Y: Down-regulation of miR-144 promotes thyroid cancer cell invasion by targeting ZEB1 and ZEB2. Endocrine 48: $566-574,2015$

20. Liu J, Xue H, Zhang J, Suo T, Xiang Y, Zhang W, Ma J, Cai D and Gu X: MicroRNA-144 inhibits the metastasis of gastric cancer by targeting MET expression. J Exp Clin Cancer Res 34: 35, 2015.

21. Iwaya T, Yokobori T, Nishidan N, Kogo R, Sudo T, Tanaka F, Shibata K, Sawada G, Takahashi Y, Ishibashi M, et al: Downregulation of miR-144 is associated with colorectal cancer progression via activation of mTOR signaling pathway. Carcinogenesis 33: 2391-2397, 2012.

22. Han S, Zhu J and Zhang Y: miR-144 potentially suppresses proliferation and migration of ovarian cancer cells by targeting RUNX1. Med Sci Monit Basic Res 24: 46-46, 2018.

23. Zhang LY, Ho-Fun Lee V, Wong AM, Kwong DL, Zhu YH, Dong SS, Kong KL, Chen J, Tsao SW, Guan XY and Fu L: MicroRNA-144 promotes cell proliferation, migration and invasion in nasopharyngeal carcinoma through repression of PTEN. Carcinogenesis 34: 454-463, 2013.

24. Ren K, Liu QQ, An ZF, Zhang DP and Chen XH: miR-144 functions as tumor suppressor by targeting PIM1 in gastric cancer. Eur Rev Med Pharmacol Sci 21: 3028-3037, 2017.

25. Livak KJ and Schmittgen TD: Analysis of relative gene expression data using real-time quantitative PCR and the 2(-Delta Delta C(T)) method. Methods 25: 402-408, 2001.

26. Alvarez RJ, Gips SJ, Moldovan N, Wilhide CC, Milliken EE, Hoang AT, Hruban RH, Silverman HS, Dang CV and Goldschmidt-Clermont PJ: 17beta-estradiol inhibits apoptosis of endothelial cells. Biochem Biophys Res Commun 237: 372-381, 1997.

27. Wu Q, Jin H, Yang Z, Luo G, Lu Y, Li K, Ren G, Su T, Pan Y, Feng $\mathrm{B}$, et al: miR-150 promotes gastric cancer proliferation by negatively regulating the pro-apoptotic gene EGR2. Biochem Biophys Res Commun 392: 340-345, 2010. 
28. Bou Kheir T, Futoma-Kazmierczak E, Jacobsen A, Krogh A Bardram L, Hother C, Grønbæk K, Federspiel B, Lund AH and Friis-Hansen L: miR-449 inhibits cell proliferation and is down-regulated in gastric cancer. Mol Cancer 10: 29, 2011.

29. Kogo R, Mimori K, Tanaka F, Komune S and Mori M: Clinical signifcance of miR-146a in gastric cancer cases. Clin Cancer Res 17: 4277-4284, 2011.

30. Tsai KW, Wu CW, Hu LY, Li SC, Liao YL, Lai CH, Kao HW, Fang WL, Huang KH, Chan WC and Lin WC: Epigenetic regulation of miR-34b and miR-129 expression in gastric cancer. Int $\mathrm{J}$ Cancer 129: 2600-2610, 2011.

31. Bai M, Li J, Yang H, Zhang H, Zhou Z, Deng T, Zhu K, Ning T, Fan $\mathrm{Q}$, Ying $\mathrm{G}$ and $\mathrm{Ba} \mathrm{Y}$ : miR-135b delivered by gastric tumor exosomes inhibits FOXO1 expression in endothelial cells and promotes angiogenesis. Mol Ther 27: 1772-1783, 2019

32. Zheng B, Yin WN, Suzuki T, Zhang XH, Zhang Y, Song LL, Jin LS, Zhan H, Zhang H, Li JS and Wen JK: Exosome-mediated miR-155 transfer from smooth muscle cells to endothelial cells induces endothelial injury and promotes atherosclerosis. Mol Ther 25: 1279-1294, 2017.

33. Gao Y, Peng J, Ren Z, He NY,Li Q, Zhao XS, Wang MM, Wen HY, Tang ZH, Jiang ZS, et al: Functional regulatory roles of microRNAs in atherosclerosis. Clin Chim Acta 460: 164-171, 2016.

34. Yamada Y, Arai T, Kojima S, Sugawara S, Kato M, Okato A, Yamazaki K, Naya Y, Ichikawa T and Seki N: Regulation of antitumor miR-144-5p targets oncogenes: Direct regulation of syndecan-3 and its clinical significance. Cancer Sci 109: 2919-2936, 2018
35. Li J, Wang R, Ge Y, Chen D, Wu B and Fang F: Assessment of microRNA-144-5p and its putative targets in inflamed gingiva from chronic periodontitis patients. J Periodontal Res 54: 266-277, 2019.

36. Wang X, Sundquist K, Hedelius A, Palmér K, Memon AA and Sundquist J: Circulating microRNA-144-5p is associated with depressive disorders. Clin Epigenetics 7: 69, 2015.

37. Zou Z, Chen J, Yang J and Bai X: Targeted inhibition of rictor/mTORC2 in cancer treatment: A new era after rapamycin. Curr Cancer Drug Targets 16: 288-304, 2016.

38. Heiss C, Rodriguez-Mateos A and Kelm M: Central role of eNOS in the maintenance of endothelial homeostasis. Antioxid Redox Signal 22: 1230-1242, 2015

39. Qin B, Shu Y, Long L, Li H, Men X, Feng L, Yang H and Lu Z: MicroR NA-142-3p induces atherosclerosis-associated endothelial cell apoptosis by directly targeting rictor. Cell Physiol Biochem 47: 1589-1603, 2018.

This work is licensed under a Creative Commons Attribution-NonCommercial-NoDerivatives 4.0 International (CC BY-NC-ND 4.0) License. 